\title{
Texture and rheological evaluation of aerated confectionery
}

\author{
Sergiu PĂDUREȚ*, Raluca - Olimpia ZIMBRU, and Sonia AMARIEI \\ Faculty of Food Engineering, Stefan cel Mare University of Suceava, Suceava, Romania
}

\begin{abstract}
Confectionery industry represents a field that uses a large number of ingredients and techniques to develop unique sweet products. To produce aerated confectionery samples two different procedures were used to incorporate the ingredients in the beating vegetable or dairy cream. The objective of this research was to determine the texture parameters and the viscoelastic properties of aerated confections using compression stress-relaxation test and applying a modified Maxwell model. The highest fat content was presented by dairy cream aerated samples (20.04-20.25\%), while the samples based on vegetable cream displayed a lower fat content. By applying the modified Maxwell mechanical model to the relaxation curves the equilibrium stress, $\sigma_{e}$, relaxation time, $\lambda_{\text {rel }}$, viscosity, $\eta$, and modulus of elasticity, $G_{0}$, were determined. The aerated samples' viscosity was greater than $137.96 \mathrm{kPa} \cdot \mathrm{s}$ and less than $451.793 \mathrm{kPa} \cdot \mathrm{s}$; furthermore, Pearson correlation showed that density influences positively this rheological parameter $\left(r=0.955^{*}\right)$. Fixing air into the product structure causes a decrease in density $\left(0.388-0.788 \mathrm{~g} / \mathrm{cm}^{3}\right)$, leading to a lower equilibrium stress, a lower elasticity modulus and also a decrease of viscosity and relaxation time.
\end{abstract}

Keywords: aerated confectionery; viscosity; relaxation time; texture; Maxwell model.

\section{Introduction}

The confectionery industry is an extremely diverse, innovative and complex food sector, with about 13000 producers throughout Europe, which manufacture both mass products and specialties [1]. The confectionery products can be classified in three main categories such as: sugar based products (hard candy, candy bars, caramels, jellies, gel foams, lollipops or nougat), chocolate products (milk chocolate, dark or white chocolate, chocolate spreads) and flour based products (cakes, shortbread cookies, wafers, salty or sweet snacks) [2, 3].

It is well known that confectionery products have a substantial caloric content, but the use of non-caloric ingredients [4], incorporation of high quantities of water and introducing air bubbles into the product's structure are some methods to lower those values [5]. Aerated foods, especially those of confectionery, constitute the height of culinary and technological mastery and deliver new and luxurious texture characteristics [6], these food products can be described in terms of porosity or pore distribution, gas content, pore or bubble dimension, density, stability and texture properties; which together offer novelty, luxury and appeal [7, 8]. Aerated food structures bring benefits during the mastication process, in the oral processing of foods, facilitate the enzyme accessibility to substrates and intensify flavors delivery [9]. The aeration process modifies also the rheological and textural characteristics of original fluid ingredients allowing them to be molding and setting into more appealing configuration [10]. According to Campbell [6] the gases of greatest importance used for aeration process in food industry are represented by air, carbon dioxide, nitrogen, nitrous oxide and steam; these gases can be used as a mixture or separately. For example, the nitrogen has applications in confectionery to produce micro-aerated structures such as those of aerated chocolate, while nitrous oxide is used as propellant gas for instant whipped cream, developing macro-aerated structures $[11,12]$. There are a few different techniques to produce aerated foods and these are based on air or gases injection, extrusion - liquid or semi solids are constrained around air/gas to form pores/bubbles, another method is represented by gases generation in the food matrix (fermentation), followed by whipping, beating, pressure beating and shaking methods $[6,10]$.

In the case of mechanical aeration methods (whipping, beating), the dimensions of air bubbles respectively the relative density of the product decrease as the speed of beating device increase, whilst a lower beating speed lead to larger air bubbles. The air bubbles' dimensions represent a primary characteristic determining its behavior and contribution to product structure and texture [13]. The aerated food system is thermodynamically unstable; the incorporated bubbles are frequently stabilized by a protein stabilizing agent or other emulsifiers which prevent the bubbles coalescence. The confectionery aerated structures are stabilized often with gelatin, egg albumin, soy proteins or dairy proteins, but other stabilizing agents such as agar, gelatinized starch, alginates, gum arabic, may also be used. Besides the role of providing stability by preventing the recombining of newly created bubbles and their coalescence, the stabilizing agent also promotes bubbles formation [14]. The texture of the aerated food products can be evaluated through different mechanical tests based on compression [15], extrusion [16] or texture profile analysis tests [8]. 
As mentioned for aerated food products, a major aspect is represented by rheological or textural evaluation and therefore the main objective of the present study is to evaluate the viscoelastic properties and texture parameters of aerated confections based on compression stress-relaxation test and Maxwell mechanical model; closely related with the physicochemical composition.

\section{Experimental}

\subsection{Materials}

The experimental aerated confectionery samples were produced through the mechanical beating process at 600 rpm [15] using milk, chocolate, sugar, yolk and dairy or vegetable cream. The sample formation followed two different procedures: the first procedure (A1 and A2 samples) consisted in homogenization of the mixture formed from beating yolk-sugar, hot milk, tempered chocolate and gelatin with beating vegetable cream (A1) and beating dairy cream (A2); the second procedure involved the incorporation in the beating vegetable cream (A3) and beating dairy cream (A4) of the other ingredients, first was added the mixture of sugar syrup and beating yolk, followed by tempered chocolate and gelatin. In order to obtain the best results, the gelatin was subjected to complete hydration $(1: 4 \mathrm{w} / \mathrm{w})$ prior to be added in the mixture, therefore it was completely active. After preparation the aerated samples were stored in closed containers at $4-6{ }^{\circ} \mathrm{C}$ until they were analyzed.

\subsection{Methods}

2.2.1. Physicochemical analysis. The moisture content of aerated samples was performed by oven assay [17] and the fat content was measured using the Soxhlet method [18]. All reagents used for physicochemical analysis were of analytical grade.

2.2.2. Textural and viscoelastic evaluation. Aerated confectionery samples with cubic shape and side of 30 mm were subjected to uniaxial compression with a Mark 10-ESM 301 Texture Analyzer equipped with $10 \mathrm{~N}$ load cell (Mark 10 Corporation, USA) using a flat probe $(\varnothing$ $50 \mathrm{~mm}$ ), the loading-unloading speed was set at 10 $\mathrm{mm} / \mathrm{min}$ and the compression distance was established at $10 \mathrm{~mm}$ [19]. The trigger force was set at $0.1 \mathrm{~N}$. After the compression distance (stress) was achieved the loading stopped and the confectionery sample was subjected to relaxation for $200 \mathrm{~s}$. During the stressrelaxation test the evolution of stress $(\mathrm{Pa})$ versus time (s) were recorded by the MESUREgauge software at a reading rate of 20 points per second (pps).

2.2.3. Modeling. The uniaxial compression stress $\left(\sigma_{0}\right.$, $\mathrm{Pa}$ ) relaxation behavior of aerated confectionery samples was described by a modified Maxwell model, formed from a single Maxwell element and a spring connected in parallel which indicates the equilibrium stress $\left(\sigma_{e}\right.$, Pa). The stress-relaxation equation (Eq. 1) describes the applied Maxwell model, where $\lambda_{\text {rel }}$ represents the relaxation time and $\left(\sigma_{0}-\sigma_{e}\right)$ represents the decay stress $\left(\sigma_{\mathrm{d}}, \mathrm{Pa}\right)[20]$ :

$$
\sigma(\mathrm{t})=\sigma_{\mathrm{e}}+\left(\sigma_{0}-\sigma_{\mathrm{e}}\right) \cdot \exp \left(\frac{-\mathrm{t}}{\lambda_{\text {rel }}}\right)
$$

Also the decay stress [21], viscosity [22] and Deborah number [23] were determined. The modified Maxwell model fits to the measured (experimental) data was achieved by the calculation of the statistical parameters such as coefficient of determination $\left(R^{2}\right)$ and absolute average deviation (AAD) (Eq. 2 and 3).

$$
\begin{gathered}
A A D=100 \frac{1}{N} \cdot \sum_{i=1}^{N}\left|\frac{y_{\mathrm{exp}, i}-y_{\text {pr }, i}}{y_{\mathrm{exp}, i}}\right| \\
R^{2}=1-\frac{\sum_{i=1}^{N}\left(y_{\mathrm{exp}, i}-y_{\text {pr }, i}\right)^{2}}{\sum_{i=1}^{N}\left(y \exp , i-y_{\text {prz, average }}\right)^{2}}
\end{gathered}
$$

Where $y_{\exp }$ and $y_{p r z}$ are the relaxation experimental (measured) and predicted values and $N$ represents the number of the experimental run [24, 25].

\subsection{Statistical analysis}

The obtain results were submitted to Pearson correlation by SPSS 13.0 (SPSS Inc. Chicago, IL).

\section{Results and discussion}

\subsection{Physicochemical analysis}

The physicochemical analysis of aerated confectionery samples is presented in Table 1 . The highest fat content was observed for dairy cream aerated samples (A2 $20.04 \%$ and A4 - 20.25\%), while the samples with vegetable cream in composition (A1 and A3) displayed a lower level of fat, ranging between $16.35 \%$ and $17.82 \%$. The moisture content of confectionery products varies considerably; it is important during the production process, represents an important storage parameter, influences the product self-life and also influences the texture characteristics [26]. The moisture content of the aerated confectionery samples varied from $42.98 \%$ to $46.42 \%$, the A1 and A2 samples having the highest concentrations. The density measurements of aerated confectionery samples are among 0.388 and $0.788 \mathrm{~g} / \mathrm{cm}^{3}$, being closely to those reported for cake batter $\left(0.55-0.80 \mathrm{~g} / \mathrm{cm}^{3}\right)$, whipped cream $(0.40-0.60$ $\left.\mathrm{g} / \mathrm{cm}^{3}\right)$ and higher than those reported for meringue $\left(0.17-0.18 \mathrm{~g} / \mathrm{cm}^{3}\right)$ and sponge cake $\left(0.25-0.35 \mathrm{~g} / \mathrm{cm}^{3}\right)$, [6]. In addition to density decreasing some studies indicates that aeration process of food matrix increases satiety reduces the energy intake [27]; food consumption being influenced by products weight and volume [5], therefore aeration satisfies consumer needs and has a potential action on obesity prevention [13].

Table 1. Texture, viscoelastic and physico-chemical parameters of aerated confectionery sample.

\begin{tabular}{|c|c|c|c|c|c|}
\hline \multicolumn{2}{|c|}{ Sample } & A1 & A2 & A3 & A4 \\
\hline Fat & {$[\%]$} & 16.35 & 20.04 & 17.82 & 20.25 \\
\hline Moisture & {$[\%]$} & 46.42 & 45.51 & 43.96 & 42.98 \\
\hline Density & {$\left[\mathrm{g} / \mathrm{cm}^{3}\right]$} & 0.388 & 0.565 & 0.760 & 0.788 \\
\hline $\begin{array}{c}\text { Equilibrium } \\
\text { stress }-\sigma_{\mathrm{e}}\end{array}$ & {$[\mathrm{kPa}]$} & 0.911 & 1.488 & 2.666 & 2.666 \\
\hline
\end{tabular}




\begin{tabular}{|c|c|c|c|c|c|}
\hline \multicolumn{2}{|c|}{ Sample } & A1 & A2 & A3 & A4 \\
\hline $\begin{array}{c}\text { Decay stress } \\
-\sigma_{d}\end{array}$ & {$[\mathrm{kPa}]$} & 0.804 & 1.483 & 1.841 & 2.347 \\
\hline $\begin{array}{c}\text { Relaxation } \\
\text { time - } \lambda_{\text {rel }}\end{array}$ & {$[\mathrm{s}]$} & 57.142 & 58.479 & 60.975 & 64.102 \\
\hline $\begin{array}{c}\text { Modulus of } \\
\text { elasticity - } \\
\mathrm{G}_{0}\end{array}$ & {$[\mathrm{kPa}]$} & 2.735 & 4.468 & 8.006 & 8.006 \\
\hline $\begin{array}{c}\text { Viscosity }-\eta \\
\text { Deborah }\end{array}$ & {$[\mathrm{kPa} \cdot \mathrm{s}]$} & 137.96 & 260.433 & 337.102 & 451.793 \\
\hline number - De & - & 0.285 & 0.292 & 0.305 & 0.320 \\
\hline
\end{tabular}

\subsection{Texture and viscoelastic evaluation}

By applying the modified Maxwell mechanical model to the relaxation curves the equilibrium stress, $\sigma_{\mathrm{e}}$, decay stress, $\sigma_{\mathrm{d}}$, relaxation time, $\lambda_{\text {rel, }}$, viscosity, $\eta$, and modulus of elasticity, $\mathrm{G}_{0}$, were determined (as shown in Table 1 ). According to Chakespari et al. [21], $\lambda_{\text {rel }}$ represents a measure of how fast a food material disperses the stress received after a sudden deformation. The relaxation time, $\lambda_{\text {rel }}$, which is a representative parameter of stress relaxation tests was greater than $57.142 \mathrm{~s}$, with a small variation up to a maximum of $64.102 \mathrm{~s}$ and $\mathrm{G}_{0}$ were about $2.735-8.006 \mathrm{kPa}$. The elastic modulus of confectionery samples was smaller than that of white pan bread or agar gel whose elastic modulus are higher than $13 \mathrm{kPa}$, but similar to those of dairy products, like ripened cheese $(0.102 \mathrm{kPa})$ [28]. Taking into account the elasticity modulus' values we can sustain that A3 and A4 samples are stiffer than A1 and A2 samples, the first procedure (which assumed the incorporation of the other ingredients' mixture at once in the beating vegetable/dairy cream) of introducing and fixing air is much better than second one. The decay stress ranged from 0.804 to $2.347 \mathrm{kPa}$, A4 samples showing the greatest decay value. It can be seen from Figure 1 that the analyzed samples exhibit a typical solid viscoelastic behavior, which implies an equilibrium stress greater than zero [28] and therefore the equilibrium stress ranged between $0.911-2.666 \mathrm{kPa}$. The viscosity observed in this study was greater than $137.96 \mathrm{kPa} \cdot \mathrm{s}$ and less than $451.793 \mathrm{kPa} \cdot \mathrm{s}$; furthermore, it showed much lower values than those reported for pears (approx. 10 $35 \mathrm{MPa} \cdot \mathrm{s}$ ) [29]. Deborah number, De, is a dimensionless parameter, which characterize the viscoelastic materials and as we can see from Table 1, the calculated De of the aerated samples was smaller than one (De $<1$ ), implying that food material exhibit more viscous than elastic behavior [20].

\subsection{Maxwell modeling}

In order to determine the viscoelastic parameters and the equations (as shown in Table 2) of the modified Maxwell model, the $\ln \left(\sigma-\sigma_{e}\right)$ versus relaxation time were represented graphically in Figure 1, the regression coefficients being higher than 0.918. Also Figure 1 presents the Maxwell model's fit (predicted data) to the measured relaxation curves and it can be established that Maxwell model with a spring connected in parallel can be successfully used to predict the viscoelastic properties of aerated confectionery samples. In addition to determination coefficient $\left(R^{2}\right)$ the absolute average deviation coefficient (AAD) was also calculated to highlight the differences between experimental data and the Maxwell model estimates. All the determination coefficients were greater than $0.9\left(0.998 \geq R^{2} \geq 0.927\right)$ and all the absolute average deviation coefficients were smaller than $3.5(2.483 \leq \mathrm{AAD} \leq 3.303)$, as shown in Table 2 and according to $[24,30]$ the higher the determination coefficients values $\left(R^{2}\right)$ and the lower the absolute average deviation coefficients values (AAD) are, the better will be the goodness of fit. Taking into account the above mentioned, the described parameters of the modified Maxwell model $\left(\sigma_{\mathrm{e}}, \sigma_{\mathrm{d}}, \lambda_{\text {rel }}, \mathrm{G}_{0}\right.$ and $\left.\eta\right)$ were at statistically appropriate levels.

Table 2. Maxwell model equations and the statistical parameters of the aerated confectionery samples

\begin{tabular}{|c|c|c|c|}
\hline Sample & Maxwell model & $\mathbf{R}^{2}$ & AAD \\
\hline A1 & $\sigma(t)=911.111+804.402 \cdot \exp \left(\frac{-t}{57.142}\right)$ & 0.939 & 2.940 \\
\hline A2 & $\sigma(t)=1488.888+1483.263 \cdot \exp \left(\frac{-t}{58.479}\right)$ & 0.927 & 3.303 \\
\hline A3 & $\sigma(t)=2666.666+1841.618 \cdot \exp \left(\frac{-t}{60.975}\right)$ & 0.998 & 2.483 \\
\hline A4 & $\sigma(t)=2666.666+2347.250 \cdot \exp \left(\frac{-t}{64.102}\right)$ & 0.962 & 2.640 \\
\hline
\end{tabular}



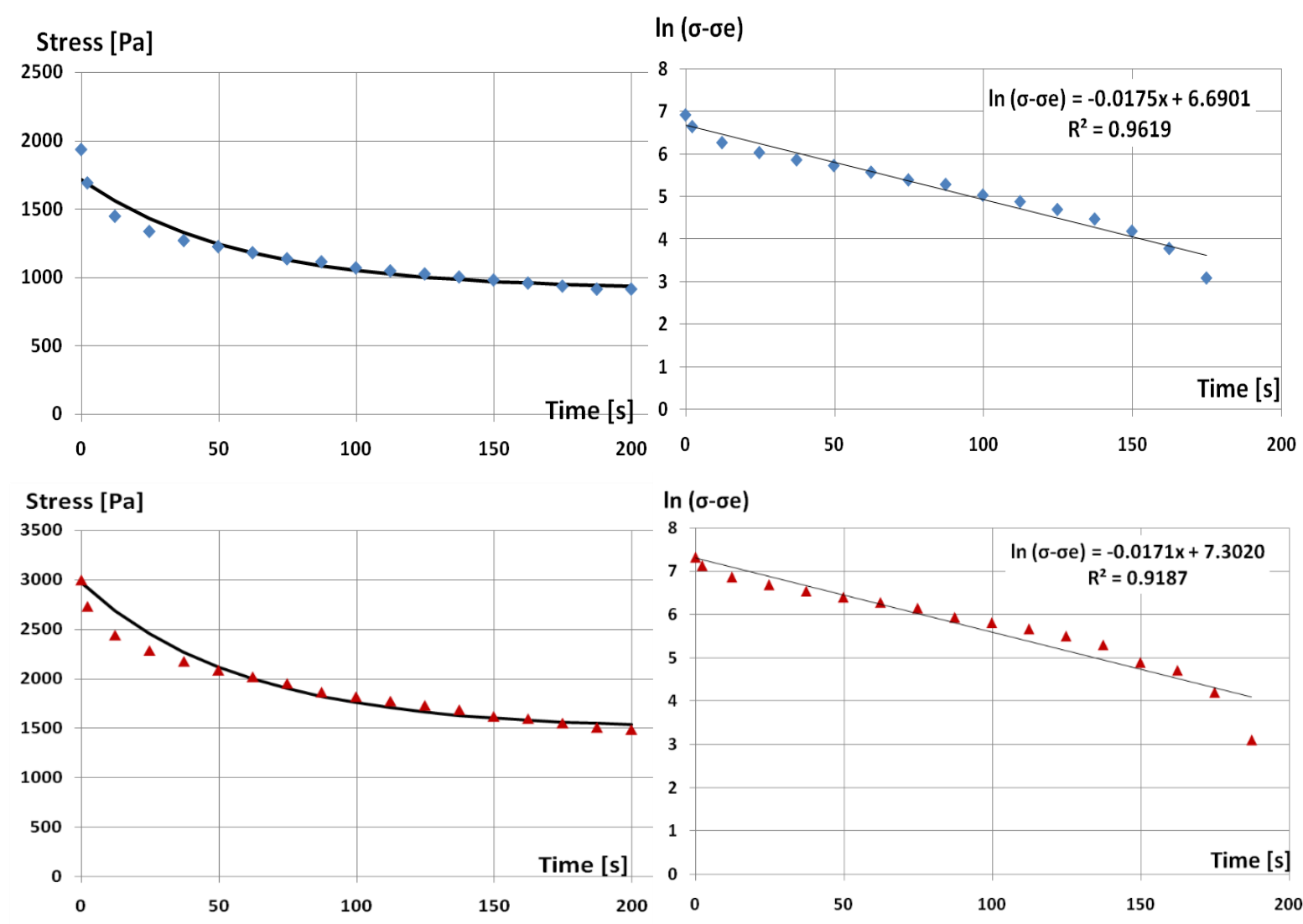

$\ln (\sigma-\sigma e)$
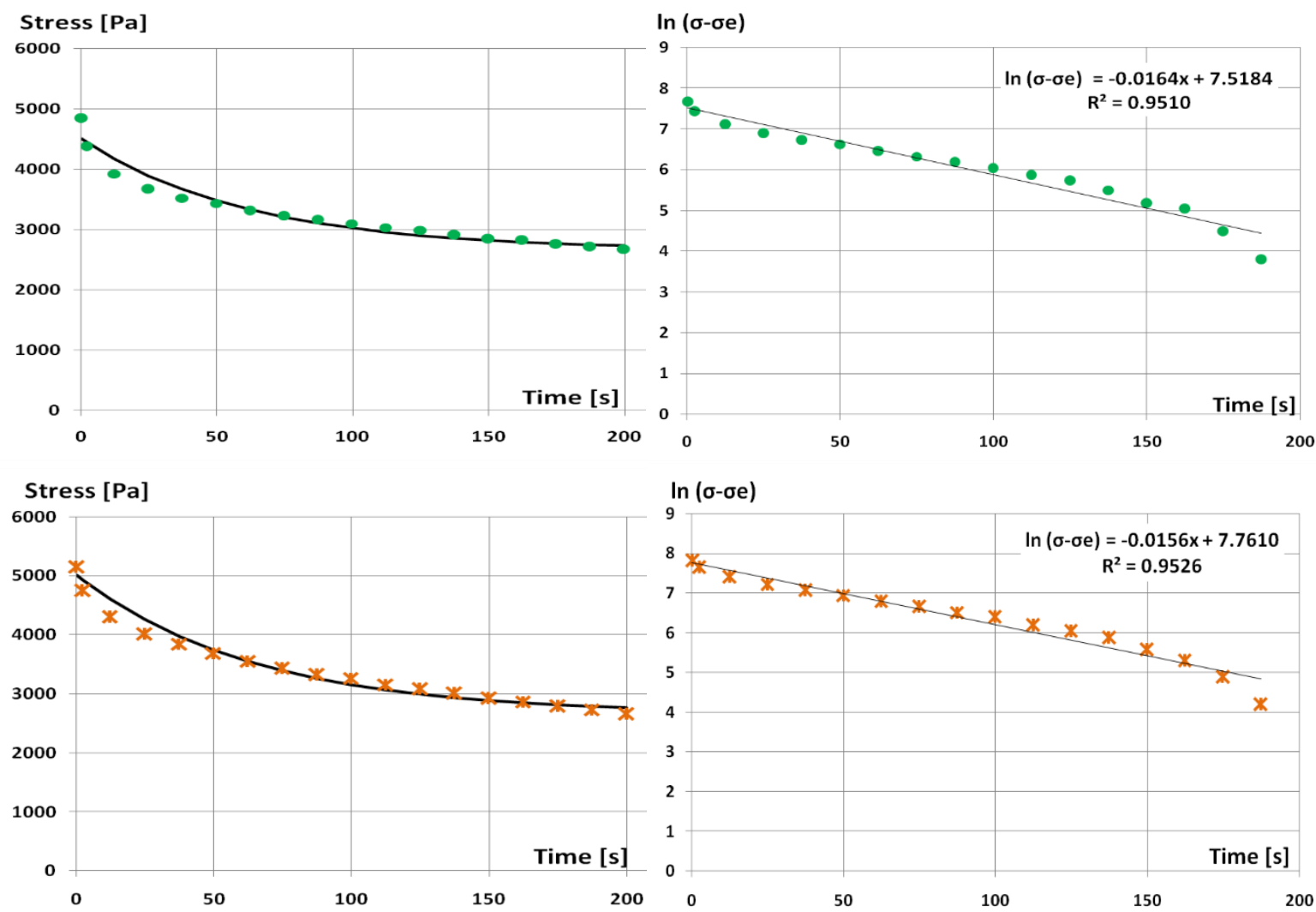

Figure 1. Relaxation curves, experimental $(\bullet, \mathbf{\Delta}, \bullet, *)$ vs. predicted data $(-)$ and $\ln (\sigma-\sigma e) v s$. relaxation time of aerated confectionery samples:A1 $(\bullet)$, A2 $(\boldsymbol{\Delta})$, A3 $(\bullet)$, A4 $(*)$.

\subsection{Pearson correlation}

Pearson correlation between physicochemical properties, texture and viscoelastic parameters of aerated confectionery samples are shown in Table 3. As regarding the physicochemical properties, a negative Pearson correlation was recorded between moisture content and aerated samples' density $\left(r=-0.932^{*}\right)$. Furthermore, moisture content influences negatively 
also the texture and viscoelastic parameters' magnitude: decay stress $\left(r=-0.990^{* *}\right)$, viscosity $\left(r=-0.984^{* *}\right)$ and relaxation time $\left(r=-0.928^{*}\right)$. According to Pearson correlation the texture and rheological parameters of aerated confectionery samples are highly positively connected between them. For aerated food products density measurement indicates the total air content embedded in the product structure [6]. The density of aerated samples influences positively texture and viscoelastic parameters like $\sigma_{e}, \sigma_{d}, \lambda_{\text {rel }}, G_{0}, \eta$, while the total air content from the product structure has a negative influences on the mentioned parameters, agreeing with [29, 31] who observed that spongy foods with an aerated structure release the stress faster.

Table 3. Pearson correlation between texture, viscoelastic and physicochemical parameters of aerated confectionery samples

\begin{tabular}{|c|c|c|c|c|c|c|c|c|c|}
\hline & $\mathrm{F}$ & $\mathrm{M}$ & $\rho$ & $\sigma_{\mathrm{e}}$ & $\sigma_{\mathrm{d}}$ & $\lambda_{\text {rel }}$ & $\mathrm{G}_{0}$ & $\eta$ & De \\
\hline $\mathrm{F}$ & 1 & -0.821 & 0.584 & 0.466 & 0.734 & 0.597 & 0.466 & 0.716 & 0.594 \\
\hline $\mathrm{M}$ & & 1 & $-0.932^{*}$ & -0.880 & $-0.990^{* *}$ & $-0.928^{*}$ & -0.880 & $-0.984^{* *}$ & $-0.930^{*}$ \\
\hline$\rho$ & & & 1 & $0.990^{* *}$ & $0.963^{*}$ & $0.915^{*}$ & $0.990^{* *}$ & $0.955^{*}$ & $0.923^{*}$ \\
\hline$\sigma_{\mathrm{e}}$ & & & & 1 & $0.930^{*}$ & $0.906^{*}$ & $1.000^{* *}$ & $0.925^{*}$ & $0.916^{*}$ \\
\hline$\sigma_{\mathrm{d}}$ & & & & & 1 & $0.963^{*}$ & $0.930^{*}$ & $0.998^{* *}$ & $0.966^{*}$ \\
\hline$\lambda_{\text {rel }}$ & & & & & & 1 & $0.906^{*}$ & $0.977^{*}$ & $1.000^{* *}$ \\
\hline $\mathrm{G}_{0}$ & & & & & & & 1 & $0.925^{*}$ & $0.916^{*}$ \\
\hline$\eta$ & & & & & & & & & $0.979^{*}$ \\
\hline $\mathrm{De}$ & & & & & & & & & 1 \\
\hline
\end{tabular}

* Correlation is significant at the 0.05 level (1-tailed). ** Correlation is significant at the 0.01 level (1-tailed).

Fat -F; Moisture -M; Density - $\rho$; Equilibrium stress - $\sigma_{e}$; Decay stress - $\sigma_{d}$; Relaxation time - $\lambda_{\text {rel }}$; Modulus of elasticity - $\mathrm{G}_{0}$; Viscosity $-\eta$; Deborah number - De

\section{Conclusions}

The two different procedures through which were produced the aerated confectionery samples had a significant effect on the texture and viscoelastic parameters. The first procedure which involved homogenization of the mixture formed from other raw materials in beating vegetable or dairy cream led to a more aerated structure of the samples (A1 and A2) with a low density and a low mechanical strength $\left(\sigma_{\mathrm{e}}\right.$ and $\left.\sigma_{\mathrm{d}}\right)$, while the incorporation of the raw materials one at a time in the beating vegetable or dairy cream (second procedure - A3 and A4) led to a higher density, a less aerated structure and also a higher mechanical strength and respectively a higher relaxation time. Taking into account the statistical parameters such as $R^{2}$ and ADD we can conclude that the modified Maxwell model can be used successfully for the estimation of the aerated confectionery's viscoelastic parameters. According to Pearson correlation $\sigma_{\mathrm{d}}, \lambda_{\text {rel }}$ and $\eta$ of aerated confectionery samples are strongly influenced by moisture content and density.

Conflicts of interest. The authors declare no conflict of interest.

Acknowledgments. This paper has been financially supported within the project entitled „DECIDE Development through entrepreneurial education and innovative doctoral and postdoctoral research, project code POCU / 380/6/13/125031, project co-financed from the European Social Fund through the 2014-2020 Operational Program Human Capital”.

\section{References}

[1]. J.H. Miah, A. Griffiths, R. McNeill, S. Halvorson, U. Schenker, N.D. Espinoza-Orias, J. Sadhukhan, Environmental management of confectionery products: Life cycle impacts and improvement strategies, Journal of Cleaner Production 177 (2018) 732-751.

[2]. W.P. Edwards, The science of sugar confectionery, Royal Society of Chemistry, Essex, UK 2018.

[3]. M. Misniakiewicz, Consumers' Expectations and Behavior towards Confectionery Products. Polish Market Case Study. 18th International Joint Conference Central and Eastern Europe in the Changing Business Environment (2018) 222-232.

[4]. M. Nastaj, B.G. Sołowiej, K. Terpiłowski, S. Mleko, Effect of erythritol on physicochemical properties of reformulated high protein meringues obtained from whey protein isolate, International Dairy Journal 105 (2020) 104672.

[5]. J.C. Arboleya, M. García-Quiroga, D. Lasa, O. Oliva, A. Luis-Aduriz, Effect of highly aerated food on expected satiety, International Journal of Gastronomy and Food Science 2 (2014) 14-21.

[6]. G.M. Campbell, Aerated foods. In: Encyclopedia of Food and Health, Elsevier Inc, United Kingdom 2016, pp. 51-60.

[7]. G. M. Campbell, A history of aerated foods, In Bubbles in Food 2, AACC International Press., U.S.A. 2008, pp. 1-21.

[8]. R.O. Zimbru, S. Pădureţ, A. Amariei, Effect of aeration on physicochemical, color and texture characteristics of confectionery foams, Ukrainian Food Journal 9 (2020) 99-110.

[9]. H. Koç, C.J. Vinyard, G.K. Essick, E.A. Foegeding, Food oral processing: conversion of food structure to textural perception, Annual 
review of food science and technology 4 (2013) 237-266.

[10]. G.M. Campbell, E. Mougeot, Creation and characterisation of aerated food products, Trends in Food Science \&Technology 10 (1999) 283-296.

[11]. J. Haedelt, S.T. Beckett, K. Niranjan, Bubbleincluded chocolate: relating structure with sensory response, Journal of Food Science 72 (2007) E138-E142.

[12]. R.N.Zúñiga, J.M. Aguilera, Aerated food gels: fabrication and potential applications, Trends in Food Science \&Technology 19 (2008) 176-187.

[13]. G. Narsimhan, Aerated Foods: Principles, Formation and Stability. John Wiley \& Sons, U.S.A. 2019.

[14]. R.W. Hartel, H. Joachim, R. Hofberger, Aerated Confections, In: Confectionery Science and Technology, Springer, Cham 2018, pp. 301-327.

[15]. G.A. Van Aken, Aeration of emulsions by whipping, Colloids and Surfaces A: Physicochemical and Engineering Aspects 190 (2001) 333-354.

[16]. H. Dogan, P.A. Romero, S. Zheng, A.M. Cuitino, J.L. Kokini, Characterization and prediction of the fracture response of solid food foams, In: Bubbles in Food 2, AACC International Press. 2008, pp. 163-174.

[17]. S.S. Nielsen (Ed.), Food analysis New York: Springer, 2010, pp. 139-141.

[18]. AOAC International, Official methods of analysis, 20th Edn., AOAC International, Rockville, MD, 2016.

[19]. G. Gutt, S. Pădureţ, S. Amariei, M. Plesca, Physical and texture parameters used in the analysis of meat freshness, Journal of Agroalimentary Prosesses and Technologies 20 (2014) 257-262.

[20]. S. Sahin, S.G Sumnu, Physical properties of foods, Springer Science \& Business Media, U.S.A. 2006.

[21]. A.G. Chakespari, A. Rajabipour, H. Mobli, Anisotropic relaxation and creep properties of apple (cv. Shafi Abadi and Golab
Kohanz), Advance Journal of Food Science and Technology, 2 (2010) 200-205.

[22]. M.A. Rao, Introduction: Food rheology and structure. In Rheology of Fluid, Semisolid, and Solid Foods Springer, Boston, MA, 2014, pp. 1-26.

[23]. R.J. Poole, The Deborah and Weissenberg numbers, The British Society of Rheology, Rheology Bulletin 53 (2012) 32-39.

[24]. S. Karaman, M.T. Yilmaz, H. Cankurt, A. Kayacier, O. Sagdic, Linear creep and recovery analysis of ketchup-processed cheese mixtures using mechanical simulation models as a function of temperature and concentration, Food Research International 48 (2012) 507-519.

[25]. S. Pădureţ, Influence of maturity stages and variety on viscoelastic properties and mechanical toughness of the strawberries, Ukrainian Food Journal 8 (2019) 733-744.

[26]. R. Ergun, R. Lietha, R.W. Hartel, Moisture and shelf life in sugar confections, Critical Reviews in Food Science and Nutrition 50 (2010) 162-192.

[27]. K.M. Osterholt, L.S. Roe, B.J. Rolls, Incorporation of air into a snack food reduces energy intake, Appetite 48 (2007) 351-358.

[28]. M.A. Del Nobile, S. Chillo, A. Mentana, A. Baiano, Use of the generalized Maxwell model for describing the stress relaxation behavior of solidlike foods, Journal of Food Engineering 78 (2007) 978-983.

[29]. J. Wang, Anisotropic relaxation properties of pear, Biosystems Engineering 85 (2003) 59-65.

[30]. H. Toğrul, N. Arslan, Mathematical model for prediction of apparent viscosity of molasses, Journal of Food Engineering 62 (2004) 281-289.

[31]. J. Tang, M.A. Tung, Y. Zeng, Characterization of gellan gels using stress relaxation, Journal of Food Engineering 38 (1998) 279- 295.

Received: 09.05.2020

Received in revised form: 28.06.2020

Accepted: 29.06.2020 